\title{
Bosonization in $2+1$ dimensions via Chern-Simons bosonic particle-vortex duality
}

\author{
Oğuz Türker, ${ }^{1}$ Jeroen van den Brink, ${ }^{1,2}$ Tobias Meng ${ }^{1},{ }^{1}$ and Flavio S. Nogueira $\odot^{2}$ \\ ${ }^{1}$ Institute for Theoretical Physics and Würzburg-Dresden Cluster of Excellence \\ ct.qmat-Complexity and Topology in Quantum Matter, TU Dresden, 01069 Dresden, Germany \\ ${ }^{2}$ Institute for Theoretical Solid State Physics, IFW Dresden, Helmholtzstraße 20, 01069 Dresden, Germany
}

(Received 4 May 2020; accepted 4 August 2020; published 17 August 2020)

\begin{abstract}
Dualities provide deep insight into physics by relating two seemingly distinct theories. Here we consider a duality between lattice fermions and bosons in $(2+1)$ spacetime dimensions, relating free massive Dirac fermions to Abelian Chern-Simons Higgs (ACSH) bosons. To establish the duality, we represent the exact partition function of the lattice fermions in terms of the writhe of fermionic worldlines. On the bosonic side, the partition function is expressed in the writhe of the vortex loops of the particle-vortex dual of the ACSH Lagrangian. In the continuum and scaling limit, we show these to be identical. This result can be understood from the closed fermionic worldlines being direct mappings of the ACSH vortex loops, with the writhe keeping track of particle statistics.
\end{abstract}

DOI: $10.1103 /$ PhysRevD.102.034506

\section{INTRODUCTION}

A duality transformation relates two theories that appear to be very different. Such a mapping is particularly useful if a seemingly hard question in one theory duality-transforms into a simple one in another theory. Duality transformations, for example, often invert the coupling constant in the dual theory, thereby transforming strongly interacting models into weakly interacting ones and vice versa [1,2]. In other cases, the transformation of the coupling constant is not a simple inversion, but rather a more complex function of the original one. Analyzing the properties of this function typically still allows one to obtain results that would be difficult to achieve otherwise. A well-known example is provided by the two-dimensional Ising model, whose dual model is again an Ising model. In this case, the self-duality allows an exact determination of the critical temperature by just looking for the fixed point of the duality transformation, a result obtained before Onsager derived the exact solution of the model [3].

Of particular interest are dualities that implement a transmutation of particle statistics in addition to a mapping of coupling constants. In one-dimensional quantum systems $(1+1$ dimensions), such transmuting mappings between fermionic theories and bosonic ones are well known and go under the general name of bosonization. Due to the

Published by the American Physical Society under the terms of the Creative Commons Attribution 4.0 International license. Further distribution of this work must maintain attribution to the author(s) and the published article's title, journal citation, and DOI. Funded by SCOAP ${ }^{3}$. fermionic sign structure of the wave function, the situation is, however, much more complex in higher dimensions. In recent years, there has been intense activity surrounding bosonization dualities in $2+1$ dimensions [4-14], but indeed it has turned out to be very difficult to obtain exact statements. For instance, while the free massive Dirac fermion in $1+1$ dimensions can be exactly mapped into a sine-Gordon model with a particular value of the coupling constant [15-17], a similar statement in $2+1$ dimensions is argued to hold only at the infrared stable fixed point of the dual bosonic theory. The latter is given in this case by the following Abelian Chern-Simons Higgs (ACSH) Lagrangian:

$\mathcal{L}=\frac{1}{4 \pi} \epsilon_{\mu \nu \lambda} a^{\mu} \partial^{\nu} a^{\lambda}+\left|\left(\partial_{\mu}-i a_{\mu}\right) \phi\right|^{2}-m^{2}|\phi|^{2}-\frac{\lambda}{2}|\phi|^{4}$.

A particularly simple classical soliton solution arises in the form of so-called "self-dual" Chern-Simons (CS) vortices [18], where "self-dual" here means the saturation of the Bogomolny bound [19] for the energy, achieved for certain values of the coupling constants, which generally leads to first-order differential equations for the fields rather than second-order ones. The static vortex solution obtained in this way features a nonzero angular momentum, which is a direct consequence of the CS term. It is interesting to note that a nonzero angular momentum for vortices is forbidden in the case of an ordinary Abelian Higgs model featuring a Maxwell term but sans CS term [20]. But this no-go theorem does not hold for the theory (1), as due to the CS term, the magnetic flux 
becomes the source of electric fields. A closely related result has been discussed recently in the context of an axion electrodynamics of vortices for a superconductortopological insulator structure [21]. The remarkable fact is that for the case of a CS coupling as given in Eq. (1), the total angular momentum associated with the electromagnetic field and vortex is quantized in units of $\hbar / 2$, which implies that the CS term transmutes the vortex into a fermion. Thus, this soliton solution of the classical field equations already suggests a boson-fermion transmutation required by bosonization techniques.

Historically, boson-fermion transmutation within a bosonization framework in $2+1$ dimensions was first discussed by Polyakov [22] for a model closely related to Eq. (1): namely, the $\mathrm{CP}^{N-1}$ model with a CS term. Polyakov's approach has been elaborated further in Refs. [23-25] and provides an early instance where the mapping of bosons to free massive Dirac fermions in $2+1$ dimensions is discussed.

One of the main results of this paper is to establish a correspondence between free massive Dirac lattice fermions and the bosonic particle-vortex duality of the Lagrangian [Eq. (1)] in the continuum limit using an exact representation of the partition function of Wilson fermions in terms of the writhe associated with the fermionic particle worldlines. Particle-vortex dualities for the Abelian Higgs model (sans CS term) in $2+1$ dimensions are well established in several different, but closely related approaches [2,26-29]: it consists in mapping the worldline of a particle in a system with global $U(1)$ symmetry (for instance, the $X Y$ model) to vortex lines of the Abelian Higgs model. This particle-vortex duality does not involve a change of statistics, as both sides of the duality involve bosonic fields only. But it suggests a pathway to establish the bosonization duality in $2+1$ dimensions: mapping the worldline of free massive fermions to the vortex loops of the Abelian Chern-Simons Higgs (ACSH) model [Eq. (1)]. Interestingly, the lattice form of the ACSH Lagrangian (1) can be mapped by means of an exact duality to a Lagrangian of almost the same form [30], differing from the original Lagrangian by the presence of a Maxwell term.

The bosonization duality in $2+1$ dimensions can be established exactly in the ultraviolet (UV) regime [9] and is assumed to hold only approximately in the infrared regime (IR). This makes it important to establish a correspondence between the bosonic particle-vortex duality and the bosonization duality in a way that is as exact as possible. This is not an obvious task, since integrating out the bosonic matter fields in Eq. (1) leads to a self-linking of vortex loops, which is a less obvious occurrence in free massive Dirac fermions, as first realized by Polyakov [22].

The plan of the paper is as follows: In Sec. II, we discuss the properties of the effective action of the bosonic theory, which prepares us for the identification of closed particle worldlines to vortex loops in later sections. In Sec. III, the fermionic sector of the duality will be considered in the lattice using the Wilson fermion technique. Since the theory is Gaussian, the partition function can be obtained exactly in the thermodynamic limit. However, in $2+1$ dimensions, free massive Dirac fermions exhibit a nontrivial topology which can only be unveiled via a subtle path-integral representation of the fermion determinant, $\operatorname{det}\left(\gamma^{\mu} \partial_{\mu}+m_{F}\right)$ $[22,31]$. In fact, despite the absence of interactions with a gauge field, the topologically nontrivial feature associated with the parity anomaly is already apparent from a straightforward exact calculation of the current correlation function. On the lattice, we determine the fermion partition function by means of a hopping parameter expansion in a way similar to Ref. [32]. This allows an exact representation of the partition function as a summation over the weights of the all possible fermion worldlines, which are closed loops characterized by the writhe number. The way the writhe arises here is a consequence of the interplay between parity symmetry breaking (due to the mass) and the nontrivial topology of spinors in $2+1$ dimensions. In Sec. IV, we discuss the convergence of the hopping expansion and cast the partition function in a form more appropriate to relate to the bosonic dual partition function. The latter is discussed in Sec. V, where the duality transformation will be performed exactly on the lattice, where in the dual model the CS coupling is inverted. The bosonization duality implies that any side of the particle-vortex duality can in principle be mapped to the Lagrangian of a free massive Dirac fermion. This fact necessarily constraints the CS coupling to have the form given in Eq. (1). We emphasize here the important role of the writhe number that naturally emerges when analyzing vortex loops in CS theories [33]. In Sec. V, we show that the partition function of the dual ACSH theory is given as the summation of the weight of all possible vortex loop configurations, where we characterize the weight of the vortex loops in terms of the writhe of the loops. Finally, in Sec. VI, the bosonization duality is established in the low-energy vortex sector.

\section{BOSONIC PARTICLE-VORTEX DUALITY, AND PROPERTIES OF THE BOSONIC CONTINUUM ACTIONS}

We begin by analyzing the (purely bosonic) particlevortex duality of the ACSH Lagrangian. For a general CS coupling $\theta / \pi$, the duality takes the form

$$
\begin{gathered}
\mathcal{L}_{b}=\frac{i \theta}{4 \pi^{2}} \epsilon_{\mu \nu \lambda} a^{\mu} \partial^{\nu} a^{\lambda}+\left|\left(\partial_{\mu}-i a_{\mu}\right) \phi\right|^{2} \\
+m_{\mathrm{B}}^{2}|\phi|^{2}+\frac{\lambda}{2}|\phi|^{4} \\
\text { 业 }
\end{gathered}
$$




$$
\begin{aligned}
\tilde{\mathcal{L}}_{b}= & \frac{1}{2 e^{2}}\left(\epsilon_{\mu \nu \lambda} \partial^{\nu} b^{\lambda}\right)^{2}+\frac{i \theta_{\mathrm{D}}}{4 \pi^{2}} \epsilon_{\mu \nu \lambda} b^{\mu} \partial^{\nu} b^{\lambda} \\
& +\left|\left(\partial_{\mu}-i b_{\mu}\right) \tilde{\phi}\right|^{2}+\tilde{m}_{\mathrm{B}}^{2}|\tilde{\phi}|^{2}+\frac{\tilde{\lambda}}{2}|\tilde{\phi}|^{4},
\end{aligned}
$$

where $\theta_{D}=-4 \pi^{4} / \theta$. Unlike the free fermionic action

$$
\mathcal{L}_{f}=\bar{\psi}\left(\gamma^{\mu} \partial_{\mu}+m_{\mathrm{F}}\right) \psi,
$$

the bosonic fields in Eq. (2) are interacting. A duality between Eqs. (2) and (3) can therefore only hold in a regime in which amplitude fluctuations of the bosonic fields are suppressed. This section establishes when this is the case, and what the properties of the bosonic continuum actions in Eq. (2) are in this regime. This will be particularly helpful in Sec. V, where we rigorously demonstrate the duality in Eq. (2) on the lattice. The results of this section will allow us to clearly identify the parameters of the lattice actions in terms of the parameters of the continuum actions.

Equation (2) subscribes to the context of a standard bosonic particle-vortex duality [2,26-29], which has also been discussed in the presence of topological terms in the past $[30,34,35]$. The dual Lagrangian $\tilde{\mathcal{L}}_{b}$ features a complex disorder field $\tilde{\phi}$ whose coupling to the gauge field $b_{\mu}$ leads to superconducting vortex lines representing the worldlines of the particles of the Lagrangian $\mathcal{L}_{b}$. In the limit $\theta \rightarrow \infty$, the bosonic particle-vortex duality (2) reduces to the well-known duality between the $X Y$ model and a superconductor described by an Abelian Higgs model $[26,28,29]$. On the other hand, for $e^{2} \rightarrow \infty$, both Lagrangians have the same form, with the CS term having inverted signs, reflecting the self-duality of the CS Abelian Higgs model with its time-reversed partner. The gauge coupling $e^{2}$ in Eq. (2b) is given by the bare phase stiffness of the Lagrangian of Eq. (2a). This statement will be made more precise in Sec. V.

If one adds a Maxwell term $\left(\epsilon_{\mu \nu \lambda} \partial_{\nu} a_{\lambda}\right)^{2} /\left(2 g^{2}\right)$ to the Lagrangian (2a) as an UV regulator, it is well known that for $\theta=0$ the charge-neutral Wilson-Fisher fixed point becomes unstable, and that the charged IR stable fixed point (sometimes called the gauged Wilson-Fisher fixed point in the more recent literature $[4,31]$ ) is perturbatively inaccessible for a single complex scalar [36-38]. The CS term makes the charged fixed point perturbatively accessible provided the RG calculations are performed using a massive scalar field, while there are indications that conformality is lost if one studies the RG flow for the critical theory [39].

To gain intuition about the role played by the CS term, one can, for example, compute the properties of the dual model (2b) (featuring a regulating Maxwell term) for a fixed uniform scalar field background, $\tilde{\phi}=\tilde{\phi}_{0}$. Integrating out the gauge field then yields

$$
\begin{aligned}
\hat{U}_{\text {eff }}\left(\tilde{\phi}_{0}\right) & =\tilde{m}_{B}^{2}\left|\tilde{\phi}_{0}\right|^{2}+\frac{\tilde{\lambda}}{2}\left|\tilde{\phi}_{0}\right|^{4}+\frac{1}{V}\left\{\sum_{\alpha= \pm} \ln \operatorname{det}\left[-\Delta+M_{\alpha}^{2}\left(\tilde{\phi}_{0}\right)\right]-\ln \operatorname{det}\left(-\Delta+2 e^{2}\left|\tilde{\phi}_{0}\right|^{2}\right)-\ln \operatorname{det}(-\Delta)\right\} \\
& =\tilde{m}_{B}^{2}\left|\tilde{\phi}_{0}\right|^{2}+\frac{\tilde{\lambda}}{2}\left|\tilde{\phi}_{0}\right|^{4}+\frac{1}{2 \pi^{2}} \int_{0}^{\Lambda} d p p^{2}\left\{\sum_{\alpha= \pm} \ln \left[1+\frac{M_{\alpha}^{2}\left(\tilde{\phi}_{0}\right)}{p^{2}}\right]-\ln \left(1+\frac{2 e^{2}\left|\tilde{\phi}_{0}\right|^{2}}{p^{2}}\right)\right\}
\end{aligned}
$$

where $V$ is the (infinite) volume, $\Lambda$ is a UV cutoff, and

$$
M_{ \pm}^{2}\left(\tilde{\phi}_{0}\right)=2 e^{2}\left|\tilde{\phi}_{0}\right|^{2}+\frac{\delta^{2}}{2} \pm \frac{|\delta|}{2} \sqrt{\delta^{2}+8 e^{2}\left|\tilde{\phi}_{0}\right|^{2}}
$$

where $\delta=e^{2} \theta_{D} /\left(2 \pi^{2}\right)$. A Landau expansion of the above effective potential up to $\left|\tilde{\phi}_{0}\right|^{4}$ yields

$$
\begin{aligned}
\tilde{U}_{\mathrm{eff}}\left(\tilde{\phi}_{0}\right) \approx & \left(\tilde{m}_{B}^{2}+\frac{\Lambda e^{2}}{\pi^{2}}-\frac{3 \sqrt{2} e^{4}}{|\theta|}\right)\left|\tilde{\phi}_{0}\right|^{2} \\
& +\frac{\sqrt{2} e}{3 \pi}\left|\tilde{\phi}_{0}\right|^{3}+\frac{\tilde{\lambda}}{2}\left|\tilde{\phi}_{0}\right|^{4}
\end{aligned}
$$

implying that the one-loop photon bubble diagram at zero external momenta gives no correction to the renormalized coupling $\tilde{\lambda}_{R}=\tilde{\lambda}+$ (quantum corrections) [39]. This is in stark contrast with the theory where a CS term is absent, where the same diagram is IR divergent and thus cannot be evaluated for zero external momenta [38]. This fact creates a difficulty to smoothly interpolate between the Abelian CS Higgs model and the standard Abelian Higgs model [39]. Given these considerations, in order to avoid the difficulties associated with the critical theory, we will assume in this paper that the fixed point structure is governed by a theory with a nonzero renormalized mass $m_{R}$ for the theory (2) [or $\tilde{m}_{R}$ for the theory (2a)], and that the IR fixed point is approached as $m_{R} \rightarrow 0$.

An interesting question is the role of amplitude fluctuations in Eq. (2b). If the scalar field $\phi_{0}$ were in a fixed, homogenous configuration, the analogue effective potential $U_{\text {eff }}\left(\phi_{0}\right)$ could easily be obtained from Eq. (4) by replacing the background field $\tilde{\phi}_{0}$ there with $\phi_{0}$, replacing $\theta_{D}$ with $\theta$, and letting $e^{2} \rightarrow \infty$. We then obtain 


$$
\begin{aligned}
U_{\mathrm{eff}}\left(\phi_{0}\right)= & m_{B}^{2}\left|\phi_{0}\right|^{2}+\frac{\lambda}{2}\left|\phi_{0}\right|^{4} \\
& +\frac{1}{2 \pi^{2}} \int_{0}^{\Lambda} d p p^{2} \ln \left[1+\frac{M^{2}\left(\phi_{0}\right)}{p^{2}}\right],
\end{aligned}
$$

where $M^{2}\left(\phi_{0}\right)=16 \pi^{4}\left|\phi_{0}\right|^{4} / \theta^{2}$. By performing the integral explicitly and assuming $\Lambda \gg|M|$, we obtain

$$
\begin{aligned}
U_{\text {eff }}\left(\phi_{0}\right) \approx & m_{B}^{2}\left|\phi_{0}\right|^{2}+\frac{1}{2}\left(\lambda+\frac{16 \pi^{2} \Lambda}{\theta^{2}}\right)\left|\phi_{0}\right|^{4} \\
& -\frac{32 \pi^{5}}{3|\theta|^{3}}\left|\phi_{0}\right|^{6}+\mathcal{O}\left(\frac{1}{\Lambda}\right) .
\end{aligned}
$$

Thus, in contrast with the standard Higgs model in $2+1$ dimensions (i.e., with a Maxwell term and without a CS term) [36], the effective potential above appears to be unstable (unbounded below) due to the generation of a negative $\left|\phi_{0}\right|^{6}$. However, since its coefficient is dimensionless and thus independent of the cutoff, it can be safely neglected. A more elaborate argument would be to notice that quite generally for a local field theory with no more than two derivatives in the classical action a $|\phi|^{6}$ term is an irrelevant operator, with the corresponding coupling constant flowing to zero anyway. Hence, we could easily absorb the constant term into a bare $|\phi|^{6}$ and set it to zero.

Thus, after dealing with the negative $|\phi|^{6}$ contribution, we see that the effective potential resembles a standard Landau theory, with $\lambda$ receiving a large shift proportional to the UV cutoff $\Lambda$. We see that even if one starts with $\lambda=0$, a scalar field self-interaction $\sim \Lambda / \theta^{2}$ is generated. We find therefore that the value of the order parameter corresponding to the minimum of the effective potential [Eq. (8)] is attained for $m_{B}^{2}<0$ and depends on $\theta$ :

$$
\left|\phi_{0, \min }\right|^{2}=-\frac{m_{B}^{2} \theta^{2}}{16 \pi^{2} \Lambda+\theta^{2} \lambda},
$$

which trivially reduces to the usual mean-field Landau theory result for $|\theta| \rightarrow \infty$. Note that one can use the UV scale $\Lambda$ to define dimensionless quantities out of both bare parameters $m_{B}^{2}$ and $\lambda$, as is customary in RG theory [15]. Thus, we would have $\lambda=\Lambda \hat{\lambda}$, where $\hat{\lambda}$ is dimensionless. The coupling constant $\lambda$ can only be disregarded in Eq. (9) if $\theta^{2} \hat{\lambda} \ll 16 \pi^{2}$.

So far, we have not considered the vortices of the theory, which are connected with the phase of the field $\phi$. This motivates us to parametrize the complex scalar field in terms of an amplitude and a phase as $\phi=\rho e^{i \varphi} / \sqrt{2}$, such that the Lagrangian of Eq. (2) becomes

$$
\begin{aligned}
\mathcal{L}_{b}= & i \frac{\theta}{4 \pi^{2}} \epsilon_{\mu \nu \lambda} a_{\mu} \partial_{\mu} a_{\lambda}+\frac{\rho^{2}}{2}\left(\partial_{\mu} \varphi-a_{\mu}\right)^{2} \\
& +\frac{1}{2}\left(\partial_{\mu} \rho\right)^{2}+\frac{m_{B}^{2}}{2} \rho^{2}+\frac{\lambda}{8} \rho^{4} .
\end{aligned}
$$

After performing the gauge transformation $a_{\mu} \rightarrow a_{\mu}+\partial_{\mu} \varphi$ and accounting for the periodic character of $\varphi$, we integrate out $a_{\mu}$ exactly to obtain

$$
\begin{aligned}
S_{\text {eff }}= & \frac{1}{2} \operatorname{Tr} \ln \left(-i \frac{\theta}{2 \pi^{2}} \epsilon_{\mu \nu \lambda} \partial_{\lambda}+\rho^{2} \delta_{\mu \nu}\right)-\frac{1}{2} \operatorname{Tr} \ln \rho^{2} \\
& +\frac{\theta^{2}}{4 \pi^{4}} \int d^{3} x \int d^{3} x^{\prime} D_{\mu \nu}\left(x, x^{\prime}\right) V_{\mu}(x) V_{\nu}\left(x^{\prime}\right) \\
& +\int d^{3} x\left[\frac{1}{2}\left(\partial_{\mu} \rho\right)^{2}+\frac{m_{B}^{2}}{2} \rho^{2}+\frac{\lambda}{8} \rho^{4}\right],
\end{aligned}
$$

where $D_{\mu \nu}\left(x, x^{\prime}\right)$ is the inverse of the operator $-i \frac{\theta}{2 \pi^{2}} \epsilon_{\mu \nu \lambda} \partial_{\lambda}+\rho^{2} \delta_{\mu \nu}$ and

$V_{\mu}(x)=\epsilon_{\mu \nu \lambda} \partial_{\nu} \partial_{\lambda} \varphi(x)=2 \pi \sum_{a} n_{a} \oint d y_{\mu}^{a} \delta^{3}\left(x-y^{a}\right)$

is the vortex loop current, with $n_{a}$ being the vortex quantum. The term (1/2) $\operatorname{Tr} \ln \rho^{2}$ arises from the Jacobian of the transformation from complex fields to $\phi=\rho e^{i \varphi} / \sqrt{2}$. It cancels out against explicit calculation of the first term of Eq. (11) in the unitary gauge [40].

For later use in the analysis of the duality using a lattice model, we integrate out the amplitude fluctuations approximately at one-loop order. This is easily done by considering the Gaussian fluctuations around $\rho_{0}=2\left|\phi_{0 \text {,min }}\right|$ in the effective action [Eq. (11)]; i.e., we consider $\rho=\rho_{0}+\delta \rho$ and integrate out the Gaussian fluctuations in $\delta \rho$. The result adds the following contribution to the effective action:

$$
\delta S_{\text {eff }}=\frac{1}{2} \operatorname{Tr} \ln \left(-\Delta+m_{B}^{2}+\frac{3 \lambda}{2} \rho_{0}^{2}\right) .
$$

A more accurate result would involve replacing $\rho_{0}^{2}$ in the above equation with $\left\langle\rho^{2}\right\rangle$ [15], and even more precise is to consider the full response and have the phase stiffness $\rho_{s}$ appearing as a coefficient of $\left(\partial_{\mu} \varphi-a_{\mu}\right)^{2}$. It is now instructive to recall a well-known random path representation for the above result $[2,27,41]$. In this case, we write the cutoff in terms of the shortest element of the path, $a$, which we later identify to be the lattice spacing, so we can write $\Lambda=\pi / a$. Denoting $P(\tilde{L})$ as the number of closed paths of length $\tilde{L}$, we can use the known results of Refs. $[2,27]$ to write

$$
\delta S_{\text {eff }}=-\int_{0}^{\infty} d L P(\tilde{L}) e^{-\epsilon \tilde{L}}
$$


where

$$
\epsilon=\frac{a}{6}\left(m_{B}^{2}+\frac{3 \lambda}{2} \rho_{0}^{2}\right)+\frac{\ln 6}{a} .
$$

The particle-vortex duality will map the particles' closed paths to vortex loops. We can use the well-known general expression for the partition function for a statistical ensemble of vortex loops as derived from particles' random worldlines [2,27]:

$$
Z=\sum_{N=0}^{\infty} \frac{1}{N !} \prod_{j=1}^{N} \sum_{\left\{C_{j}\right\}} e^{-S_{\text {vortex }}-\epsilon \tilde{L}\left(C_{j}\right)},
$$

where $S_{\text {vortex }}$ is the action yielding the (long-range) interaction energy between two loops $C_{i}$ and $C_{j}, \tilde{L}\left(C_{j}\right)$ is the length of the $j$ th loop, and here $\epsilon$ is identified as the vortex core energy $[2,42]$.

\section{WILSON FERMIONS IN THREE- DIMENSIONAL EUCLIDEAN SPACETIME}

In this section, we determine the partition function of a free massive Dirac theory in a three-dimensional cubic Euclidean lattice. In order to put the Dirac theory into the lattice, we use Wilson fermions [43]; see also Ref. [44]. This results in lattice action for the free massive Dirac fermions:

$$
\begin{aligned}
S= & a^{2}\left[\left(m_{0} a+3 R\right) \sum_{n} \bar{\psi}_{n} \psi_{n}-\frac{1}{2} \sum_{n \mu}\left[\bar{\psi}_{n}\left(R-\gamma_{\mu}\right) \psi_{n+\hat{\mu}} U_{\mu n}\right.\right. \\
& \left.\left.+\bar{\psi}_{n+\hat{\mu}}\left(R+\gamma_{\mu}\right) \psi_{n} U_{\mu n-\hat{\mu}}^{\dagger}\right]\right]
\end{aligned}
$$

where $R$ is the Wilson parameter and $a$ is the lattice spacing, which we set to unity until otherwise specified. The Euclidean Dirac matrices above are given by the Pauli matrices.

In the continuum limit, Eq. (17) will converge to Eq. (3) independent of the value of the $R$ as long as $R \neq 0$. We also assume that $m_{0}>0$. The coupling of the Wilson fermions to the field $U_{\mu n}=\exp \left(-i A_{\mu, n}\right)$ enforces the local gauge invariance of Eq. (17) in the lattice. Rewriting Eq. (17) as in Ref. [32], we find

$$
\begin{gathered}
S=\frac{1}{2 \kappa} \sum_{n m} \bar{\psi}_{n} K_{n m}[U] \psi_{m}, \\
K[U]_{n m}=\delta_{n m} \mathbb{1}-\kappa M_{n m}[U], \\
M_{n n+\hat{\mu}}[U]=\left(R-\gamma_{\mu}\right) U_{\mu n}, \\
M_{n n-\hat{\mu}}[U]=\left(R+\gamma_{\mu}\right) U_{\mu n-\hat{\mu}}^{\dagger},
\end{gathered}
$$

where $\kappa=\frac{1}{2\left(m_{0}+3 R\right)}$ and Eqs. (18c) and (18d) give nonzero elements of $M$. This leads to the lattice partition function

$$
\begin{aligned}
Z & =\int \mathcal{D} \bar{\psi} \mathcal{D} \psi e^{-\frac{1}{2 \kappa} \sum_{n m} \bar{\psi}_{n} K_{n m}[U] \psi_{m}} \\
& =\operatorname{det}\left[\frac{1}{2 \kappa} K[U]\right] \\
& =\exp \left[-\sum_{n=1}^{\infty} \frac{\kappa^{2 n}}{2 n} \operatorname{Tr}\left[M^{2 n}[U]\right]\right],
\end{aligned}
$$

where we have dropped a constant term $\exp [-\operatorname{Tr}[\ln (2 \kappa)]]$ in the last step. We only sum over even numbers of products of $M$ 's, since the trace of an odd number of $M$ 's vanishes, as is apparent from Eqs. (18c) and (18d). The convergence of this series is explicitly discussed in Sec. IV.

In the next steps, we will calculate $\operatorname{Tr}\left[M^{2 n}\right]$. Being a trace, only paths forming closed loops contribute. Thus,

$$
Z=\exp \left[-\sum_{n=1} \sum_{\left\{C_{2 n}\right\}} \frac{\kappa^{2 n}}{I\left(C_{2 n}\right)} \operatorname{tr}\left[M_{C_{2 n}}\right]\right],
$$

where the trace symbol $\operatorname{tr}[\ldots]$ is over products of Pauli matrices and should not be confused with $\operatorname{Tr}[\ldots]$, which denotes the trace over spacetime indices in the lattice. $M_{C_{2 n}}$ is a $2 n$-fold path-ordered product of $M$ 's along a path $C_{2 n}$ consisting of $2 n$ number of lattice sites. From now on, we choose the value of the Wilson parameter to be $R=1$. One main advantage of this choice is that now summation will be over all possible connected, nonbacktracking paths of length $2 n$, with $I\left(C_{2 n}\right)$ being the number of times a fermion travels along the path $C_{2 n}$. Thus, $I\left(C_{2 n}\right)$ plays the role of a winding number. Simple examples of loops are illustrated in Fig. 1. Backtracking paths like the one arising in the loop of Fig. 1(d) yield no contribution when $R=1$, since in this case $R \pm \gamma_{\mu}$ are projection operators and we have $\left(1-\gamma_{\mu}\right)\left(1+\gamma_{\mu}\right)=0$. This special choice of $R$ is irrelevant in the scaling limit implied by the continuum model, which corresponds to the regime where $m_{0} a \ll 1$.

From this point forward, we take $A_{\mu n}=0$ and define the projector

$$
\Gamma(\vec{e}(i))=1-\vec{\gamma} \cdot \vec{e}(i)
$$

where $\vec{e}(i)$ is a unit vector tangent to an $i$ th segment of a given path. Thus,

$$
\operatorname{tr}\left[M_{C_{2 n}}\right]=\operatorname{tr}\left[\prod_{i=1}^{2 n} \Gamma(\vec{e}(i))\right],
$$

yielding [32] 
(a)

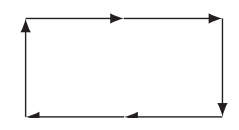

(c)

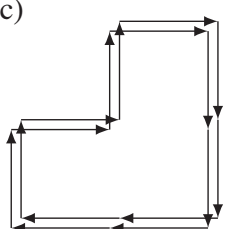

(b)

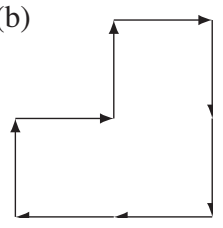

(d)

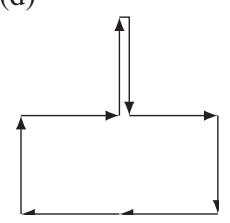

FIG. 1. (a) For this loop, the perimeter $L$ is equal to the number of lattice sites $2 n=6$, the number of straight sections/sides is $k=4$, and the number of windings $I=1$. (b) Here, $2 n=8$, $k=6$, and $I=1$ (the number of winding would not change with the orientation of the loop). (c) Here, $2 n=16, I=2$, and $k=12$.

(d) Nonallowed loop, since it consists of backtracking.

$$
\begin{aligned}
\operatorname{tr}\left[\prod_{i=1}^{2 n} \Gamma(\vec{e}(i))\right]= & \operatorname{tr}\left[\prod_{i=1}^{k} \Gamma(\vec{e}(i))^{p_{k}}\right] \text { with } \vec{e}(i) \neq \vec{e}(i+1) \\
= & 2^{2 n-k} \operatorname{tr}\left[\prod_{i=1}^{k} \Gamma(\vec{e}(i))\right] \text { with } \\
& \vec{e}(i) \neq \vec{e}(i+1)
\end{aligned}
$$

where $k$ is the number of straight sections (or sides) of a given loop, $p_{k}$ is the length of the $k$ th straight section, and in the last equality we use $\Gamma(\vec{e}(i))^{n}=2^{n-1} \Gamma(\vec{e}(i))$.

Next, we parametrize the unit tangent vectors in spherical coordinates as

$$
\vec{e}(i)=\left(\sin \theta_{i} \cos \phi_{i}, \sin \theta_{i} \sin \phi_{i}, \cos \theta_{i}\right)
$$

and represent the eigenstates of the vector of Pauli matrices as $|\vec{e}(i) ; \pm\rangle$, which satisfy

$$
\vec{\gamma} \cdot \vec{e}(i)|\vec{e}(i) ; \pm\rangle= \pm|\vec{e}(i) ; \pm\rangle,
$$

implying in this way

$$
\Gamma(\vec{e}(i))=2|\vec{e}(i) ;-\rangle\langle\vec{e}(i) ;-| .
$$

Therefore,

$$
\operatorname{tr}\left[\prod_{i=1}^{k} \Gamma(\vec{e}(i))\right]=2^{k} \prod_{i=1}^{k}\langle\vec{e}(i) ;-\mid \vec{e}(i+1) ;-\rangle,
$$

where $|\vec{e}(k+1) ;-\rangle=|\vec{e}(1) ;-\rangle$. From now on, we denote $|\vec{e}(i) ;-\rangle \equiv|\vec{e}(i)\rangle$ for notational simplicity. The inner product can be written in terms of amplitude and phase, as

$$
\begin{aligned}
& \langle\vec{e}(i) \mid \vec{e}(i+1)\rangle \\
& \quad=|\langle\vec{e}(i) \mid \vec{e}(i+1)\rangle| \exp (i \arg [\langle\vec{e}(i) \mid \vec{e}(i+1)\rangle]) .
\end{aligned}
$$

Note that $|\langle\vec{e}(i) \mid \vec{e}(i+1)\rangle|=\frac{1}{\sqrt{2}}$ for all $i$, which can be seen by simply calculating the magnitude of the inner product between all distinct pairs. For the phase factor, we have

$$
\begin{aligned}
\arg \langle\vec{e}(i) \mid \vec{e}(i+1)\rangle & =\arctan \left[\frac{\sin \Delta \phi_{i} \cot \frac{\theta_{i}}{2} \cot \frac{\theta_{i+1}}{2}}{1+\cos \Delta \phi_{i} \cot \frac{\theta_{i}}{2} \cot \frac{\theta_{i+1}}{2}}\right] \\
& =\frac{\Omega_{i}^{\prime}}{2},
\end{aligned}
$$

where $\Omega_{i}^{\prime}$ is the area of a spherical triangle on a unit sphere $[45,46]$, which is shown in Fig. 2 with the corners defined by the unit vectors $-\hat{z}, \vec{e}(i)$, and $\vec{e}(i+1)$. Now, if we combine Eq. (29) with Eq. (27), we obtain

$$
\begin{aligned}
\operatorname{tr}\left[\prod_{i=1}^{k} \Gamma(\vec{e}(i))\right] & =2^{k / 2} \prod_{i=1}^{k} \exp \left(i \frac{\Omega_{i}^{\prime}}{2}\right) \\
& =2^{k / 2} \exp i \frac{\Omega^{\prime}}{2}
\end{aligned}
$$

Note that $\vec{e}$ is a unit tangent to the path $C_{2 n}$. Now, if we consider a moving frame on path $C_{2 n}$, we define $\tilde{C}$ as the path such that the tip of $\vec{e}$ draws in this moving frame; see Fig. 3. As is shown in Fig. 2, if we define $\Omega$ as the total solid angle acquired by $\vec{e}$ while traveling the given loop, then $\Omega^{\prime}=4 \pi-\Omega$. This can be better seen if we assume for a moment a continuous case such that $\Delta \phi_{i} \ll 1$ and $\theta_{i} \sim \theta_{i+1}$. In this case, we would have

$$
\Omega_{i}^{\prime} \sim \Delta \phi_{i}\left(1+\cos \theta_{i}\right)
$$

and thus

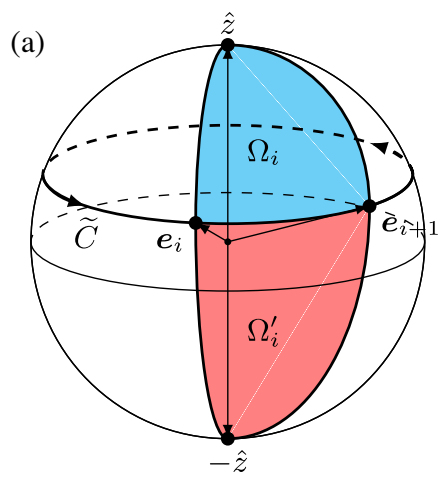

(b)

FIG. 2. For a given $\tilde{C}$, (a) $\Omega_{i}$ is the solid angle traced by $\vec{e}_{i}$ while traveling from $i$ to $i+1$. (b) $\Omega^{\prime}$ is $4 \pi-\Omega$. 
(a)

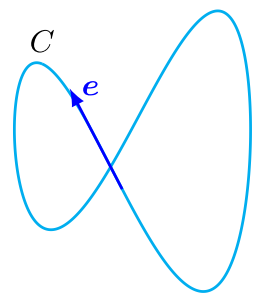

(b)

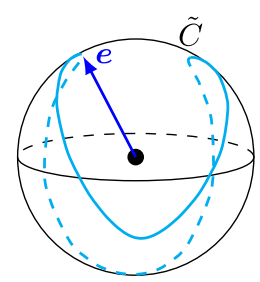

FIG. 3. (a) $C$ is the worldline of a fermion and $\vec{e}$ is the unit tangent to $C$. (b) $\tilde{C}$ is the curve drawn by the tip of $\vec{e}$ while traveling on $C$.

$$
\begin{aligned}
\exp i \frac{\Omega^{\prime}}{2} & =\exp \left[\frac{i}{2} \int_{\tilde{C}} d s \dot{\phi}(1+\cos \theta)\right] \\
& =\exp \left(\frac{i}{2}[4 \pi-\Omega]\right) \\
& =\exp \left(-\frac{i}{2} \Omega\right)
\end{aligned}
$$

Thus, after Taylor-expanding the exponential in Eq. (20), we can express the partition function as

$$
Z=\sum_{N=0}^{\infty} \frac{1}{N !} \prod_{j}^{N}\left[\sum_{\left\{C_{j}\right\}}-2^{L\left[C_{j}\right]-k_{j} / 2} \frac{\kappa^{L\left[C_{j}\right]}}{I_{C_{j}}} e^{-\frac{i}{2} \Omega_{C_{j}}}\right],
$$

where $\sum_{\left\{C_{j}\right\}}$ is summation over all nonbacktracking connected loops and $L\left[C_{j}\right]$ is the perimeter of the loop, which is simply given by the number of sites on the loop. In Eq. (33), we sum over all possible loops, where some of these loops trace a path several times, as is shown in Fig. 1(c). We can rewrite Eq. (33) in an equivalent form where we sum over loops with single winding as

$Z=\sum_{N=0}^{\infty} \frac{1}{N !} \prod_{j}^{N}\left[\sum_{\left\{C_{j}\right\}^{\prime}} \sum_{n_{j}=1}^{\infty}-2^{n_{j} L\left[C_{j}\right]-\frac{n_{j} k_{j}}{2}} \frac{\kappa^{n_{j} L\left[C_{j}\right]}}{n_{j}} e^{-\frac{i}{2} n_{j} \Omega_{C_{j}}}\right]$,

where $\sum_{\left\{C_{j}\right\}^{\prime}}$ denotes the sum over loops with single windings and $n_{j}$ is the winding number.

Finally, we can write the solid angle $\Omega$ swept out by $\vec{e}$ in terms of the writhe of the curve. To do so, we follow the approach of Refs. [23,47]: Assume that we have a closed curve $C_{1}$ of length $L$ with parametrization $\vec{r}_{1}(s)$ where $0 \leq s \leq L$. We also set $s$ as the arc length between $\vec{r}_{1}(0)$ and $\vec{r}_{1}(s)$ of the curve, which implies $\left|\dot{\vec{r}}_{1}\right|=1$. Now, assume that we have another curve $C_{2}$, with parametrization $\vec{r}_{2}$, such that $\vec{r}_{2}=\vec{r}_{1}+\epsilon \vec{a}$, where $\vec{a}$ is the unit vector normal to $\vec{r}_{1}$; i.e., $\vec{r}_{1} \cdot \vec{a}=0$, and $\epsilon$ is an infinitesimal constant; see Fig. 4. Now, recall the Călugăreanu-White theorem [48-50], which relates and defines the linking number $G$, the writhe $\mathcal{W}$, and the twist $T$ of two curves as $[23,47]$ (a)

(b)
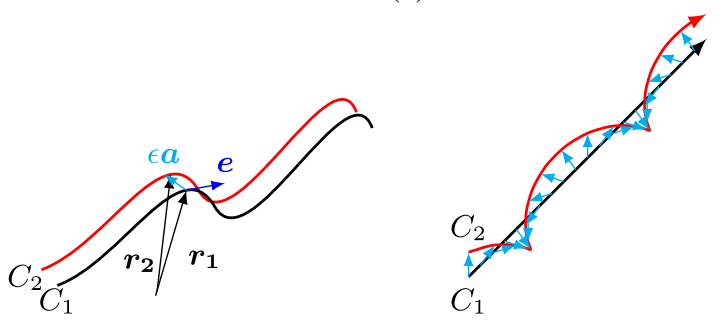

FIG. 4. (a) Here $\vec{e}$ is the tangent vector at point $\vec{r}_{1}$ on curve $C_{1}$, and $\epsilon \vec{a}$ is the distance between $C_{2}$ and $C_{1}$, where $\vec{a} \cdot \vec{r}_{1}=0$. (b) If $C_{2}$ winds around $C_{1}, \vec{a}$ rotates around $C_{1}$. If we view $C_{1}$ and $C_{2}$ as edges of a ribbon, then $\vec{a}$ rotates as the ribbon twists. The expression $\frac{\vec{a} \times \vec{a} c^{2}}{\epsilon^{2}} \cdot \vec{e}$ can be regarded as the angular speed of the point $\epsilon \vec{a}$ around $\vec{e}$, and the expression for the twist as given in Eq. (35d) is the angular displacement of $\epsilon \vec{a}$ divided by $2 \pi$.

$$
\begin{gathered}
G\left[C_{1}, C_{2}\right]=\mathcal{W}\left[C_{1}\right]+T\left[C_{1}, C_{2}\right], \\
G\left[C_{1}, C_{2}\right]=\frac{1}{4 \pi} \oint_{C_{1}} \oint_{C_{2}} \frac{d \vec{r}_{1} \times d \vec{r}_{2} \cdot\left[\vec{r}_{1}-\vec{r}_{2}\right]}{\left|\vec{r}_{1}-\vec{r}_{2}\right|^{3}}, \\
\mathcal{W}\left[C_{1}\right]=\frac{1}{4 \pi} \oint_{C_{1}} \oint_{C_{1}} \frac{d \vec{r}_{1} \times d \vec{r}_{2} \cdot\left[\vec{r}_{1}-\vec{r}_{2}\right]}{\left|\vec{r}_{1}-\vec{r}_{2}\right|^{3}}, \\
T\left[C_{1}, C_{2}\right]=\frac{1}{2 \pi} \int_{0}^{L} d s[\vec{a}(s) \times \vec{a}(s)] \cdot \vec{r}_{1}(s) .
\end{gathered}
$$

We now seek to relate the writhe $\mathcal{W}$ to $\Omega$, and we proceed by first relating the twist $T$ to $\Omega$ and then using Eq. (35a). Since $\left|\dot{\vec{r}}_{1}\right|=1, \dot{\vec{r}}_{1}$ is the unit tangent vector to the curve $C_{1}$-i.e., $\dot{\vec{r}}_{1}(s)=\vec{e}(s)$-and we parametrize it as $\vec{e}(s)=\hat{\vec{e}}_{r}(s)$, where $\hat{\vec{e}}_{r}(s)$ is a radial unit vector in spherical coordinates as in Eq. (24), and we can choose the frame vector [23] $\vec{a}(s)=\hat{\vec{e}}_{\phi}(s)$, where $\hat{\vec{e}}_{\phi}(s)$ is the azimuthal unit vector in spherical coordinates. We then evaluate Eq. (35d) as

$$
\begin{aligned}
T\left[C_{1}, C_{2}\right] & =\frac{1}{2 \pi} \oint_{C_{1}} d \vec{r}_{1} \cdot[\vec{a}(s) \times \overrightarrow{\dot{a}}(s)] \\
& =\frac{1}{2 \pi} \int_{0}^{L} d s \dot{\phi}(s) \cos \theta(s) .
\end{aligned}
$$

Next, the solid angle traced by $\hat{\vec{e}}_{r}$ while traveling on $C_{1}$ is given as

$$
\begin{aligned}
\Omega & =\oint_{\tilde{C}_{1}} d \vec{e}_{r} \cdot \hat{\vec{e}}_{\phi} \frac{1-\cos \theta}{\left|e_{r}\right| \sin \theta} \\
& =\int_{0}^{L} d s \dot{\phi}(s)(1-\cos \theta(s)) \\
& =2 \pi u-\int_{0}^{L} d s \dot{\phi}(s) \cos \theta(s), \quad u \in \mathbb{Z} \\
& =-2 \pi T\left[C_{1}, C_{2}\right] \quad \bmod (2 \pi) .
\end{aligned}
$$


Note that in Eq. (37) we take the line integral over a vector potential of a Dirac monopole along the curve $\tilde{C}_{1}$, which yields the solid angle associated with $\tilde{C}_{1}$, which corresponds to the solid angle swept by $\vec{e}$ traveling along $C_{1}$. In Eq. (37c), we use the fact that $\vec{r}_{1}(0)=\vec{r}_{1}(L)$. Finally, we substitute Eq. (37d) into Eq. (35a) to get

$$
\mathcal{W}[C]=\frac{\Omega}{2 \pi}+p,
$$

where $p=G-u$. Finally, we can use the observation that $p$ is an odd integer [23].

Now, by putting Eq. (38) into Eq. (34), we obtain the exact fermionic partition function as a series in $\kappa$ of the form

$$
\begin{array}{r}
Z=\sum_{N=0}^{\infty} \frac{1}{N !} \prod_{j}^{N}\left[\sum_{\left\{C_{j}\right\}^{\prime}} \sum_{n_{j}=1}^{\infty} z\left[C_{j}, n_{j}\right]\right], \\
z=-2^{n_{j} L\left[C_{j}\right]-\frac{n_{j} k_{j}}{2}} \frac{\kappa^{n_{j} L\left[C_{j}\right]}}{n_{j}}(-1)^{n_{j}} e^{-i \pi n_{j} \mathcal{W}\left[C_{j}\right]},
\end{array}
$$

where $C_{j}$ denotes a closed loop with straight sections $k_{j}$, length $L\left[C_{j}\right]$, winding number $n_{j}$, and-most importantlywrithe $\mathcal{W}\left[C_{j}\right]$.

As a side note, the above result is closely related to the usual loop representation of the fermionic determinant in terms of loops, within a so-called hopping parameter expansion (more details can be found in Sec. 5.1.3 of Ref. [51]).

\section{CONVERGENCE OF THE LATTICE FERMIONIC DETERMINANT}

The series expansion for the lattice determinant for Wilson fermions above in Eq. (19c) raises the question as to its radius of convergence, and in particular the critical value of $\kappa$. For this discussion we adopt the approach of Ref. [52]. Since the term in the exponential of Eq. (19c) is the series expansion of $\operatorname{Tr}[\ln [1-\kappa M]]$, the series converges for

$$
\kappa|| M \|_{\infty}<1,
$$

where the infinity norm is given as the square root of the largest [53] eigenvalue of $M^{\dagger} M$ [52]. In order to find it, we first use the Fourier representation of $M$,

$$
\begin{aligned}
M_{n m} & =\sum_{\mu} \int_{-\pi}^{\pi} \frac{d^{3} p}{(2 \pi)^{3}} e^{i p(n-m)} 2\left[\cos \left(p_{\mu}\right)-i \gamma_{\mu} \sin \left(p_{\mu}\right)\right] \\
& =\int_{-\pi}^{\pi} \frac{d^{3} p}{(2 \pi)^{3}} e^{i p(n-m)} \sum_{\alpha= \pm}|\alpha\rangle\langle\alpha| E_{\alpha}(p)
\end{aligned}
$$

where in the last line we write $M$ in a diagonal form using the eigenvectors $|\alpha\rangle$ of $M_{n m}$, and $E_{ \pm}(p)=$ $2\left[\sum_{\mu} \cos \left(p_{\mu}\right) \pm i\left[\sum_{\mu} \sin ^{2}\left(p_{\mu}\right)\right]^{1 / 2}\right]$. Thus, we can Fouriertransform $M^{\dagger} M$ as

$\left(M^{\dagger} M\right)_{n m}=\int_{-\pi}^{\pi} \frac{d^{3} p}{(2 \pi)^{3}} e^{i p(n-m)} \sum_{\alpha}|\alpha\rangle\left\langle\left.\alpha|| E_{\alpha}(p)\right|^{2}\right.$,

where $|E(p)|^{2}=4\left[\left[\sum_{\mu} \cos \left(p_{\mu}\right)\right]^{2}+\sum_{\mu} \sin ^{2}\left(p_{\mu}\right)\right]$. Thus, $|E(0)|^{2}=36$ is the highest eigenvalue, and the norm is $\|M\|_{\infty}=6$. We parametrize $\kappa$ as

$$
\kappa=\frac{1}{6} e^{-m}
$$

where $m=\ln \left(1+\frac{m_{0}}{3}\right)$; then, using Eq. (40) we find that the series converges for $m_{0}>0$. Thus, not surprisingly, we find that the series converges only for a nonzero bare mass. We can now rewrite Eq. (39a) using Eq. (43) as

$$
\begin{gathered}
Z=\sum_{N=0}^{\infty} \frac{1}{N !} \prod_{j}^{N}\left(\sum_{\left\{C_{j}\right\}^{\prime}} \sum_{n_{j}=1}^{\infty} z\left[C_{j}, n_{j}\right]\right), \\
z=-\frac{2^{L\left[C_{j}\right] n_{j}-n_{j} k_{j}\left[C_{j}\right] / 2}}{6^{n_{j} L\left[C_{j}\right]}} \frac{(-1)^{n_{j}}}{n_{j}} e^{-m n_{j} L\left[C_{j}\right]} e^{-i \pi n_{j} \mathcal{W}\left[C_{j}\right]} .
\end{gathered}
$$

This final form of the fermionic partition sum expressed in terms of the writhe is our first key result.

\section{PARTICLE-VORTEX DUALITY IN THE CHERN-SIMONS LATTICE ABELIAN HIGGS MODEL}

Having studied the bosonic continuum actions of Eq. (2) in Sec. II, and having given the form of the partition sum of the fermionic action in terms of the writhe derived in the last section, we aim to establish the bosonization duality between the lattice versions of Eqs. (2) and (3) by also expressing the bosonic partition sum in terms of the writhe. We proceed in three steps: first, we connect the bosonic particle-vortex duality in the continuum to its lattice equivalent; then, we calculate the bosonic partition sum in the dual bosonic action; and finally, we compare it with the fermionic result.

\section{A. Particle-vortex duality on the lattice}

The partition function for the Abelian CS Higgs model on the lattice with a noncompact gauge field is given by

$$
Z=\left[\prod_{j, \mu} \int_{0}^{2 \pi} \frac{d \varphi_{j}}{2 \pi} \int_{-\infty}^{\infty} d a_{j \mu}\right] \sum_{\left\{n_{j \mu}\right\}} e^{-S},
$$


where the lattice action is given in the Villain approximation as

$S=\sum_{j}\left[i \frac{\theta}{4 \pi^{2}} \epsilon_{\mu \nu \lambda} a_{j \mu} \Delta_{\nu} a_{j \nu}+\frac{J}{2}\left(\Delta_{\mu} \varphi_{j}-2 \pi n_{j \mu}-a_{j \mu}\right)^{2}\right]$,

where $\sum_{\left\{n_{i \mu}\right\}}=\prod_{i \mu} \sum_{n_{i \mu}=-\infty}^{\infty}, J>0$ is the bare phase stiffness, and $\Delta_{\mu}$ represents the forward discrete derivative, $\Delta_{\mu} f_{i}=f_{i+1}-f_{i}$. In the above action, the integer-valued lattice fields $n_{j \mu}$ enforce the periodicity of $\varphi_{j}$ fulfilling the integer (or vortex) gauge invariance, $\varphi_{j} \rightarrow \varphi_{j}+2 \pi L_{j \mu}$, $n_{j \mu} \rightarrow n_{j \mu}+L_{j \mu}$, where $L_{j \mu}$ is an arbitrary integer. This discrete gauge invariance is a common feature of the socalled Villain action [2] and arises here in addition to the usual gauge invariance associated with the lattice gauge field $a_{j \mu}$.

In order to derive the dual model, we follow closely the approach of Ref. [26] and use the Poisson summation formula,

$$
(2 \pi a)^{1 / 2} \sum_{n=-\infty}^{\infty} e^{-\frac{a}{2}(x-2 \pi n)^{2}}=\sum_{m=-\infty}^{\infty} e^{-\frac{1}{2 a} m^{2}+i x m}
$$

to introduce an auxiliary integer-valued lattice field $J_{j \mu}$, i.e.,

$$
\sum_{\left\{n_{j \mu}\right\}} e^{-\frac{J}{2}\left(\Delta_{\mu} \varphi_{j}-2 \pi n_{j \mu}-a_{j \mu}\right)^{2}} \sim \sum_{\left\{J_{j \mu}\right\}} e^{-\frac{1}{2 J} J_{j \mu}^{2}+i J_{j \mu}\left(a_{j \mu}-\Delta_{\mu} \varphi_{j}\right)}
$$

Next, we use summation by parts in the term $-J_{j \mu} \Delta_{\mu} \varphi_{j}$ to convert it to $\Delta_{\mu} J_{j \mu} \varphi_{j}$, which allows us to integrate the phase variables out to obtain the zero divergence constraint, $\Delta_{\mu} J_{j \mu}=0$. The latter implies that we are dealing with a sum over configurations where the vortices form loops [2]. The action is thus rewritten as

$$
S^{\prime}=\sum_{j}\left[i \frac{\theta}{4 \pi^{2}} \epsilon_{\mu \nu \lambda} a_{j \mu} \Delta_{\nu} a_{j \nu}+\frac{1}{2 J} J_{j \mu}^{2}-i J_{j \mu} a_{j \mu}\right]
$$

The constraint is solved by introducing the curl of another integer field, $M_{j \mu}$, such that $J_{j \mu}=\epsilon_{\mu \nu \lambda} \Delta_{\nu} M_{j \lambda}$, which leads to

$$
\begin{aligned}
S^{\prime \prime}= & \sum_{j}\left[i \frac{\theta}{4 \pi^{2}} \epsilon_{\mu \nu \lambda} a_{j \mu} \Delta_{\nu} a_{j \nu}+\frac{1}{2 J}\left(\epsilon_{\mu \nu \lambda} \Delta_{\nu} M_{j \lambda}\right)^{2}\right. \\
& \left.-i\left(\epsilon_{\mu \nu \lambda} \Delta_{\nu} M_{j \lambda}\right) a_{j \mu}\right] .
\end{aligned}
$$

Thus, upon integrating out the gauge field $a_{j \mu}$, we obtain

$$
\begin{aligned}
Z & =\sum_{\left\{M_{j \mu}\right\}} e^{-S^{\prime \prime \prime}} \\
S^{\prime \prime \prime} & =\frac{1}{2} \sum_{j}\left[\frac{1}{J}\left(\epsilon_{\mu \nu \lambda} \Delta_{\nu} M_{j \lambda}\right)^{2}-i \frac{2 \pi^{2}}{\theta} \epsilon_{\mu \nu \lambda} M_{j \mu} \Delta_{\nu} M_{j \lambda}\right] .
\end{aligned}
$$

We now use the Poisson summation formula in the form

$$
\sum_{n=-\infty}^{\infty} f(n)=\sum_{m=-\infty}^{\infty} \int_{-\infty}^{\infty} \frac{d k}{2 \pi} e^{i 2 \pi k m} f(k)
$$

to convert the integer field $M_{j \mu}$ into a real-valued gauge field $b_{j \mu}$, and noting that this last step introduces another integer field $\tilde{J}_{j \mu}$, we obtain finally the dual action in the form

$$
\begin{aligned}
\tilde{S}= & \frac{1}{2} \sum_{j}\left[\frac{1}{J}\left(\epsilon_{\mu \nu \lambda} \Delta_{\nu} b_{j \lambda}\right)^{2}-i \frac{2 \pi^{2}}{\theta} \epsilon_{\mu \nu \lambda} b_{j \mu} \Delta_{\nu} b_{j \lambda}\right] \\
& -i 2 \pi \sum_{j} \tilde{J}_{j \mu} b_{j \mu},
\end{aligned}
$$

after integrating out $a_{j \mu}$. Note that unlike the original action [Eq. (46)], the dual action above features a Maxwell term with the bare phase stiffness of the original model appearing as a gauge coupling. As a consequence of gauge invariance, the lattice vortex current field $\tilde{J}_{j \mu}$ also has a vanishing divergence.

By letting $J \rightarrow \infty$ in Eq. (53), we see that for $\theta=\pi$ and after rescaling $b_{j \mu} \rightarrow b_{j \mu} /(2 \pi)$, the latter is the same as the one arising in the partition function [Eq. (49)] up to the sign of the CS term. As far as the partition function is concerned, the sign of the CS term is immaterial, since integrating out either $a_{j \mu}$ or $b_{j \mu}$ in the $J \rightarrow \infty$ limit leads to the same vortex current interaction when $\theta=\pi$ as we sum over all integer-valued vortex currents. The theory is thus self-dual in this regime.

If we smear the constraint $\Delta_{\mu} \tilde{J}_{j \mu}=0$ by adding the term $(\gamma / 2) \tilde{J}_{j \mu}^{2}$ to the dual action (53), corresponding to adding a chemical potential for the vortex loops [26,28], and apply once more the Poisson formula [Eq. (47)], we obtain

$$
\begin{aligned}
\tilde{S}^{\prime}= & \frac{1}{2} \sum_{j}\left[\frac{1}{J}\left(\epsilon_{\mu \nu \lambda} \Delta_{\nu} b_{j \lambda}\right)^{2}-i \frac{2 \pi^{2}}{\theta} \epsilon_{\mu \nu \lambda} b_{j \mu} \Delta_{\nu} b_{j \lambda}\right. \\
& \left.+\frac{1}{\gamma}\left(\Delta_{\mu} \tilde{\varphi}_{j}-2 \pi N_{j \mu}-2 \pi b_{j \mu}\right)^{2}\right]
\end{aligned}
$$

where $N_{j \mu}$ is an integer lattice field and $\tilde{\varphi}_{j}$ is a phase variable originating from the integral representation of the Kronecker delta constraint enforcing $\Delta_{\mu} \tilde{J}_{j \mu}=0$, i.e., 


$$
\delta_{\Delta_{\mu} \tilde{J}_{j \mu}, 0}=\int_{-\pi}^{\pi} \frac{d \tilde{\varphi}_{j}}{2 \pi} e^{i \tilde{\varphi}_{j} \Delta_{\mu} \tilde{J}_{j \mu}}
$$

Equation (10), Eq. (46), and its dual form in Eq. (54) establish a lattice version of the field theory duality of Eq. (2) in the regime where amplitude fluctuations of the bosonic fields are negligible. The continuum limit of the lattice duality is expected to approach the field theory duality in the vicinity of the critical point. However, we should emphasize that precise statements to this effect can only be achieved within the lattice formalism.

\section{B. Duality and writhe}

In the case of the partition function for Wilson fermions, we have seen that in order to make the writhe more apparent, we had to partially evoke a continuum limit while still counting fermionic loop configurations. We will employ a similar strategy for the dual bosonic partition function (53) in order to express it terms of linking and writhe numbers. Thus, the continuum version of Eq. (53) can be obtained by first writing a functional integral for a given configuration featuring $N$ vortex loops:

$$
\begin{aligned}
& Z \propto \sum_{\left\{\tilde{J}_{x \mu}\right\}}{ }^{\prime} \int \mathcal{D} b e^{-S_{\mathrm{MCS}},} \\
& S_{\mathrm{MCS}}=\int d^{3} x\left[\frac{1}{2 J}\left(\epsilon_{\mu \nu \lambda} \partial_{\nu} b_{\lambda}\right)^{2}\right. \\
&\left.-\frac{i \pi^{2}}{\theta} \epsilon_{\mu \nu \lambda} b_{\mu} \partial_{\nu} b_{\lambda}-i 2 \pi \tilde{J}_{\mu} b_{\mu}\right],
\end{aligned}
$$

with

$$
\tilde{J}_{\mu}(x)=\sum_{a=1}^{N} n_{a} \int_{0}^{\tilde{L}_{a}} d s \frac{d y_{\mu}^{a}(s)}{d s} \delta^{3}\left(x-y^{a}(s)\right),
$$

where $n_{a} \in \mathbb{Z}^{+}[54]$ is the quantum number of the $a$ th vortex loop, and $y_{\mu}^{a}(s), s \in\left[0, \tilde{L}_{a}\right]$ is a parametrization of the curve describing a loop $C_{a}$ with length $\tilde{L}_{a}$ satisfying the boundary conditions $y_{\mu}^{a}(0)=y_{\mu}^{a}\left(\tilde{L}_{a}\right)$. Equation (57) clearly satisfies $\partial_{\mu} \tilde{J}_{\mu}=0$.

We can write the partition function as a summation over all possible vortex current field configurations $\tilde{J}$ as

$$
Z=\sum_{N=0}^{\infty} \frac{1}{N !} \prod_{j=1}^{N} \sum_{\left\{C_{j}\right\}^{\prime}} \sum_{n_{j}} Z\left(C_{1}, n_{1} ; \ldots ; C_{N}, n_{N}\right),
$$

where $Z\left(C_{1}, n_{1} ; \ldots ; C_{N}, n_{N}\right)$ is a partition function with a fixed vortex configuration which consists of $N$ number of vortex loops, where the $a$ th loop has a shape $C_{a}$ and vortex quantum number $n_{a}$. Multiplication by $\frac{1}{N !}$ provides the symmetry factor preventing the overcounting of identical configurations, and $\sum_{\{C\}^{\prime}}$ is summation over all connected nonbacktracking loops with single winding and with both orientations. Explicitly, we can write the partition function for a fixed configuration as

$$
Z\left(C_{1}, n_{1} ; \ldots ; C_{N}, n_{N}\right)=\int \mathcal{D} b_{\mu} e^{-S_{\mathrm{MCS}}},
$$

normalized such that $Z(0)=1$. We now integrate out $b_{\mu}$, in momentum space so that

$$
\begin{aligned}
& Z\left(C_{1}, n_{1} ; \ldots ; C_{N}, n_{N}\right) \\
& \quad=\exp \left[-2 \pi^{2} \int \frac{d^{3} p}{(2 \pi)^{3}} D_{\mu \nu}(p) \tilde{J}_{\mu}(p) \tilde{J}_{\nu}(-p)\right],
\end{aligned}
$$

where

$D_{\mu \nu}(p)=\frac{J}{p^{2}+4 \pi^{4} J^{2} / \theta^{2}}\left(\delta_{\mu \nu}-\frac{p_{\mu} p_{\nu}}{p^{2}}-\frac{2 \pi^{2} J}{\theta} \frac{\epsilon_{\mu \nu \lambda} p_{\lambda}}{p^{2}}\right)$

in the Landau gauge. Equation (60) gives a vortex interaction identical to the one in Eq. (11) when $\rho$ is uniform.

In the limit where $J \rightarrow \infty$, which corresponds to $\Lambda \rightarrow \infty$ in the case of $\rho_{0}$, we obtain

$$
\begin{aligned}
& Z\left(C_{1}, n_{1} ; \ldots ; C_{N}, n_{N}\right) \\
& \quad=\exp \left[\frac{i \theta}{4 \pi} \sum_{a, b} n_{a} n_{b} \oint_{C_{a}} \oint_{C_{b}} d y_{\mu}^{a} d y_{\nu}^{b} \frac{\epsilon_{\mu \nu \lambda}\left(y_{\lambda}^{a}-y_{\lambda}^{b}\right)}{\left|y^{a}-y^{b}\right|^{3}}\right],
\end{aligned}
$$

which in view of Eq. (35) can be rewritten as

$$
Z(\{C\})=\exp \left(i \theta \sum_{a} n_{a}^{2} \mathcal{W}\left[C_{a}\right]+i 2 \theta \sum_{a<b} n_{a} n_{b} G_{a b}\right),
$$

where we have introduced the notation $\{C\}=\left\{C_{1}, n_{1} ; \ldots\right.$; $\left.C_{N}, n_{N}\right\}$. For $\theta=\pi$, the term proportional to $G_{a b}$ does not contribute to the partition function due to the Gauss linking number theorem. If we use a statistical mechanical language and interpret $J$ as the exchange energy divided by the temperature and refer to the original lattice action [Eq. (46)], we see that the limit $J \rightarrow \infty$ corresponds to a zero-temperature limit in this context. Furthermore, $J$ is related to the amplitude of the scalar field $\phi$, such that $J \sim \rho_{0}^{2}$, and we have seen in Sec. II that $\rho_{0}^{2}$ is indeed very large. Peskin [26] in his analysis of the particle-vortex duality referred to this regime as a "frozen superconductor." The analysis in Ref. [26] ignores the vortex core energy and adds it by hand as a small chemical potential for the vortices $[26,28]$. However, the vortex core energy arises quite naturally, since it is related to the correlation length in 
the continuum theory. Furthermore, there is a direct relation between it and the bare mass, as we discussed in Sec. II. Therefore, the actual result corresponding to large $J$ is given by

$$
\begin{aligned}
& Z(\{C\}) \\
& \quad=\exp \left(i \theta \sum_{a} n_{a}^{2} \mathcal{W}\left[C_{a}\right]+i 2 \theta \sum_{a<b} n_{a} n_{b} G_{a b}-\epsilon \sum_{a} n_{a}^{2} \tilde{L}_{a}\right),
\end{aligned}
$$

where $\epsilon$ is the vortex core energy given by Eq. (15). As in Eq. (63), for $\theta=\pi$ the second term does not contribute, since $G_{a b} \in \mathbb{Z}$ by virtue of the Gauss linking number theorem. Thus, after summing over all loop configurations, we obtain the partition function as

$$
Z=\sum_{N=0}^{\infty} \frac{1}{N !} \prod_{j=1}^{N} \sum_{\left\{C_{j}\right\}} \sum_{n_{j}}\left[\exp \left(i \pi \mathcal{W}\left[C_{j}\right]\right) \exp \left(-\epsilon \tilde{L}\left[C_{j}\right]\right)\right]^{n_{j}^{2}},
$$

an equation that has the same form as Eq. (16), with $S_{\text {vortex }}=-i \pi \mathcal{W}$. The factor $\exp \left(i \pi \mathcal{W}\left[C_{j}\right]\right)$ is crucial for the fermion-boson transmutation in $2+1$ dimensions and is referred to as a "spin factor" in the literature $[25,31,55]$.

\section{COMPARISON OF FERMIONIC AND BOSONIC PARTITION FUNCTIONS}

Let us now compare the fermionic partition function with the bosonic one in the continuum. In order to take the continuum limit of the lattice fermionic partition function, we first return to Eq. (27) and introduce the lattice spacing explicitly:

$$
\begin{aligned}
\operatorname{tr}\left[M_{C_{2 n}}\right] & =\operatorname{tr}\left[\prod_{j=1}^{2 n} \Gamma(\vec{e}(j a))\right]=\prod_{j=1}^{2 n} 2\langle\vec{e}(j a) \mid \vec{e}((j+1) a)\rangle \\
& =\prod_{j=1}^{2 n} 2\left[1+\left.a\left\langle\vec{e}(s) \mid \frac{d}{d s} \vec{e}(s)\right\rangle\right|_{s=j a}+\mathcal{O}\left(a^{2}\right)\right] \\
& =\prod_{j=1}^{2 n} 2\left[1+\frac{i}{2} a \dot{\phi}(s)(1+\cos (\theta(s)))+\mathcal{O}\left(a^{2}\right)\right] \\
& \sim 2^{\frac{\tilde{L}}{a}} \exp -\frac{i}{2} \Omega\left[C_{\tilde{L}}\right],
\end{aligned}
$$

where $\tilde{L}[C]=a L[C]$, and in the last line we use Eq. (32). In this case Eq. (44) becomes

$$
\begin{array}{r}
Z=\sum_{N=0}^{\infty} \frac{1}{N !} \prod_{j}^{N}\left[\sum_{\left\{C_{j}\right\}^{\prime}} \sum_{n_{j}=1}^{\infty} z\left[C_{j}, n_{j}\right]\right], \\
z=-2^{\tilde{L}\left[C_{j}\right] n_{j} / a} e^{-i \pi n_{j} W\left[C_{j}\right]-(m+\ln 6) \tilde{L}\left[C_{j}\right] n_{j} / a} \frac{(-1)^{n_{j}}}{n_{j}} .
\end{array}
$$

We confine ourselves to the low-energy sector corresponding to $n_{j}=1$, in which case the fermionic partition function becomes

$Z_{F}=\sum_{N=0}^{\infty} \frac{1}{N !} \prod_{j=1}^{N} \sum_{\left\{C_{j}\right\}} 2^{\tilde{L}\left[C_{j}\right] / a} e^{-i \pi W\left[C_{j}\right]-(m+\ln 6) \tilde{L}\left[C_{j}\right] / a}$.

In the limit $m_{0} a \ll 1$, we have $m=\ln \left(1+m_{0} a / 3\right) \approx$ $m_{0} a / 3$, which implies

$$
Z_{F} \propto \sum_{N=0}^{\infty} \frac{1}{N !} \prod_{j=1}^{N} \sum_{\left\{C_{j}\right\}} e^{-i \pi W\left(C_{j}\right)-\tilde{M}_{0} \tilde{L}\left(C_{j}\right)}
$$

where

$$
\tilde{M}_{0}=\frac{m_{0}}{3}+\frac{1}{a} \ln 3 .
$$

The key observation is that the bosonic partition function [Eq. (65)] [56]

$$
\epsilon=\tilde{M}_{0}=\frac{m_{0}}{3}+\frac{1}{a} \ln 3 .
$$

It is possible to connect this result to the continuum theory via the ensemble of paths discussed in Sec. II. Accordingly, by using Eq. (15), we obtain a relation between the fermion, the boson mass $m_{B}$, and the $|\phi|^{4}$ coupling $\lambda$ of the continuum model:

$$
\frac{m_{0} a}{3}=\frac{a^{2}}{6}\left(m_{B}^{2}+\frac{3 \lambda}{2} \rho_{0}^{2}\right)+\ln 2 .
$$

Thus, we have found that in the regime where the vortex energy entering the Boltzmann weight is minimum, corresponding to the winding $n_{j}=1$ in Eq. (65), the latter is identical to the fermionic partition function where in the fermion loop worldlines the particle travels the loop only once. This establishes a correspondence between the fermionic particle worldline loops and vortex loops in the bosonic dual CS theory, which in turn sheds new light on Polyakov's result for a first-quantized path-integral description of massive Dirac fermions in $2+1$ dimensions [22].

\section{DISCUSSION}

The duality transformation from the Villain action [Eq. (46)] to Eq. (54) identifies the continuum limit to the duality [Eq. (2)]. In contrast to the situation in $3+1$ dimensions, the Maxwell term is IR-irrelevant in $2+1$ dimensions, and therefore both theories in Eq. (2) flow to the same gauged (IR-stable) Wilson-Fisher fixed point. This situation corresponds in the lattice to $J$ large compared to the momentum scale. In the field theory, we identify $e^{2}=J$. The renormalization of $e^{2}$ can be obtained as usual 
from the parity-even contribution to the vacuum polarization, $\Pi(p)$. Thus, the renormalized gauge coupling is given simply by

$$
e_{R}^{2}=\frac{e^{2}}{1+e^{2} \Pi(0)}
$$

Gauge invariance implies that $\Pi(0)=k / \hat{m}_{R}$, where $k$ is some universal constant. Therefore, for $e^{2} \rightarrow \infty$ we obtain that $e_{R}^{2} \approx \tilde{m}_{R} / k$. Equivalently, keeping $e^{2}$ fixed and approaching the critical point, $\tilde{m}_{R} \rightarrow 0$ yields the same scaling behavior, leading once more to $e_{R}^{2} \approx \tilde{m}_{R} / k$. Since $e^{2}$ is identified by the duality as the bare phase stiffness, the scaling behavior $e_{R}^{2} \approx \tilde{m}_{R} / k$ corresponds precisely to the Josephson scaling relation [57]. At the same time, we expect that the dimensionless renormalized coupling $\tilde{\lambda}_{R} / \tilde{m}_{R}$ approaches the (gauged) Wilson-Fisher fixed point as $\tilde{m}_{R} \rightarrow 0$.

What does the above picture imply for the Dirac fermions? It is usually conjectured that $m_{B}^{2}=0$ implies $m_{0}=0[4,5,9]$. Inserting this into Eq. (72) yields, at the lowest order based on the mean-field result [Eq. (9)],

$$
\frac{m_{0} a}{3}=\ln 2
$$

which clearly never vanishes. However, it is important to realize that the actual critical point corresponds to $m_{R}=0$, which is in general not attained for $m_{B}^{2}=0$. Furthermore, a more accurate picture should relate $m_{0}$ to the phase stiffness beyond the one-loop result. But then other complications may arise, as the fermions will presumably not be in the free theory regime any longer.

\section{CONCLUSION}

In Sec. V, we performed in the lattice an exact duality transformation mapping the partition function of bosons in the ACSH model to the partition function for an ensemble of closed vortex loop excitations of the same model, which corresponds in field theory language to the correspondence shown in the first two lines of the above equation. Furthermore, in Sec. VI, we were able to identify the partition function for an ensemble of vortex loops of the
ACSH model in the low-energy regime to the partition function for an ensemble of closed fermion worldlines.

More precisely, we have studied in this work the correspondence between the ACSH model and free massive fermions within the framework of a particle-vortex duality. This was achieved via an exact duality transformation where closed worldlines of bosonic particles arising in the partition function of the ACSH model are brought to an equivalent form by summing over an ensemble of closed vortex loops of the same model. Thanks to the CS term in the action, this standard particle-vortex duality features a phase factor in the partition function where the phase is given by the writhe number of a pair of vortex loops. We then showed that the fermionic partition function represented as a sum over an ensemble of closed paths of fermionic particles features exactly the same phase factor involving the writhe. In this case, the match between the fermionic and bosonic partition functions is established in the low-energy regime, where the mass of the fermions is naturally related to the vortex core energy. It turns out that the latter also corresponds to the energy density per element of the path of the bosons in the particle representation of the partition function.

Various aspects of this bosonization duality have been studied intensely in the past, providing conjectures as well as exact results in several limiting cases. Our calculation focuses on the self-dual point $\theta=\pi$ of the particle-vortex duality of the ACSH model and provides exact duality mappings for the lattice versions of Eqs. (2) and (3). We find that the bosonization duality holds for $\theta=\pi$ because the Gauss linking number contributions are then suppressed from the bosonic partition function, leaving only the writhe number contribution. Concretely, the bosonic dual partition function takes the form of a sum over all possible vortex loops with a given writhe yielding the phase factor mentioned above.

\section{ACKNOWLEDGMENTS}

This work is supported by the DFG through the Würzburg-Dresden Cluster of Excellence on Complexity and Topology in Quantum Matter-ct.qmat (EXC 2147, project-id No. 39085490) and through SFB 1143 (projectid No. 247310070). O. T. and T. M. furthermore acknowledge financial support by the DFG via the Emmy Noether Programme No. ME4844/1-1.
[1] R. Savit, Duality in field theory and statistical systems, Rev. Mod. Phys. 52, 453 (1980).

[2] H. Kleinert, Gauge Fields in Condensed Matter: Vol. 1: Superflow and Vortex Lines (Disorder Fields, Phase Tran- sitions) Vol. 2: Stresses and Defects (Differential Geometry, Crystal Melting) (World Scientific, Singapore, 1989).

[3] H. A. Kramers and G. H. Wannier, Statistics of the twodimensional ferromagnet: Part I, Phys. Rev. 60, 252 (1941). 
[4] N. Seiberg, T. Senthil, C. Wang, and E. Witten, A duality web in $2+1$ dimensions and condensed matter physics, Ann. Phys. (Amsterdam) 374, 395 (2016).

[5] A. Karch and D. Tong, Particle-Vortex Duality from 3D Bosonization, Phys. Rev. X 6, 031043 (2016).

[6] D. F. Mross, J. Alicea, and O. I. Motrunich, Symmetry and Duality in Bosonization of Two-Dimensional Dirac Fermions, Phys. Rev. X 7, 041016 (2017).

[7] O. Aharony, F. Benini, P.-S. Hsin, and N. Seiberg, ChernSimons-matter dualities with SO and USp gauge groups, J. High Energy Phys. 02 (2017) 72.

[8] F. Benini, Three-dimensional dualities with bosons and fermions, J. High Energy Phys. 02 (2018) 68.

[9] J.-Y. Chen, J. H. Son, C. Wang, and S. Raghu, Exact BosonFermion Duality on a 3D Euclidean Lattice, Phys. Rev. Lett. 120, 016602 (2018).

[10] Y. Ferreiros and E. Fradkin, Boson-fermion duality in a gravitational background, Ann. Phys. (Amsterdam) 399, 1 (2018).

[11] H. Nastase and C. Nez, Deriving three-dimensional bosonization and the duality web, Phys. Lett. B 776, 145 (2018).

[12] C. Wang, A. Nahum, M. A. Metlitski, C. Xu, and T. Senthil, Deconfined Quantum Critical Points: Symmetries and Dualities, Phys. Rev. X 7, 031051 (2017).

[13] S. B. Dutta and R. Shankar, Bosonization, coherent states and semiclassical quantum Hall skyrmions, J. Phys. Condens. Matter 20, 275237 (2008).

[14] R. C. B. Santos, P. R. S. Gomes, and C. A. Hernaski, Bosonization of the Thirring model in $2+1$ dimensions, Phys. Rev. D 101, 076010 (2020).

[15] J. Zinn-Justin, Quantum Field Theory and Critical Phenomena, 4th ed. (Clarendon Press, Oxford, 2002).

[16] E. Witten, Non-Abelian bosonization in two dimensions, Commun. Math. Phys. 92, 455 (1984).

[17] M. Stone, Bosonization (World Scientific, Singapore, 1994).

[18] R. Jackiw and E. J. Weinberg, Self-Dual Chern-Simons Vortices, Phys. Rev. Lett. 64, 2234 (1990).

[19] N. Manton and P. Sutcliffe, Topological Solitons (Cambridge University Press, Cambridge, England, 2004).

[20] B. Julia and A. Zee, Poles with both magnetic and electric charges in non-Abelian gauge theory, Phys. Rev. D 11, 2227 (1975).

[21] F. S. Nogueira, Z. Nussinov, and J. van den Brink, Fractional Angular Momentum at Topological Insulator Interfaces, Phys. Rev. Lett. 121, 227001 (2018).

[22] A. M. Polyakov, Fermi-Bose transmutations induced by gauge fields, Mod. Phys. Lett. A 03, 325 (1988).

[23] J. Grundberg, T. H. Hansson, A. Karlhede, and U. Lindström, Spin, statistics and linked loops, Phys. Lett. B 218, 321 (1989).

[24] N. Shaji, R. Shankar, and M. Sivakumar, On Bose-Fermi equivalence in a U(1) gauge theory with Chern-Simons action, Mod. Phys. Lett. A 05, 593 (1990).

[25] J. Grundberg, T. H. Hansson, and A. Karlhede, On Polyakov's spin factors, Nucl. Phys. B347, 420 (1990).

[26] M. E. Peskin, Mandelstam-'t Hooft duality in Abelian lattice models, Ann. Phys. (N.Y). 113, 122 (1978).

[27] P. R. Thomas and M. Stone, Nature of the phase transition in a non-linear O(2)3 model, Nucl. Phys. B144, 513 (1978).
[28] C. Dasgupta and B. I. Halperin, Phase Transition in a Lattice Model of Superconductivity, Phys. Rev. Lett. 47, 1556 (1981).

[29] H. Kleinert, Disorder version of the Abelian Higgs model and the order of the superconductive phase transition, Lett. Nuovo Cimento 35, 405 (1982).

[30] S.-J. Rey and A. Zee, Self-duality of three-dimensional Chern-Simons theory, Nucl. Phys. B352, 897 (1991).

[31] H. Goldman and E. Fradkin, Loop models, modular invariance, and three-dimensional bosonization, Phys. Rev. B 97, 195112 (2018).

[32] I. O. Stamatescu, Note on the lattice fermionic determinant, Phys. Rev. D 25, 1130 (1982).

[33] S. Forte, Quantum mechanics and field theory with fractional spin and statistics, Rev. Mod. Phys. 64, 193 (1992).

[34] J. L. Cardy and E. Rabinovici, Phase structure of ZP models in the presence of a parameter, Nucl. Phys. B205, 1 (1982).

[35] J. L. Cardy, Duality and the parameter in Abelian lattice models, Nucl. Phys. B205, 17 (1982).

[36] B. I. Halperin, T. C. Lubensky, and S.-K. Ma, First-Order Phase Transitions in Superconductors and Smectic- $a$ Liquid Crystals, Phys. Rev. Lett. 32, 292 (1974).

[37] I. D. Lawrie, On the phase transitions in Abelian Higgs models, Nucl. Phys. B200, 1 (1982).

[38] I. F. Herbut and Z. Tešanović, Critical Fluctuations in Superconductors and the Magnetic Field Penetration Depth, Phys. Rev. Lett. 76, 4588 (1996).

[39] F. S. Nogueira, J. van den Brink, and A. Sudbo, Conformality lost and quantum criticality in topological Higgs electrodynamics in $2+1$ dimensions, Phys. Rev. D 100, 085005 (2019).

[40] F. S. Nogueira and H. Kleinert, Field theoretic approaches to the superconducting phase transition, in Order, Disorder and Criticality (World Scientific, Singapore, 2004), pp. 253-283.

[41] C. Itzykson and J.-M. Drouffe, Statistical Field Theory: Volume 1, Strong Coupling, Monte Carlo Methods, Conformal Field Theory and Random Systems, Vol. 1 (Cambridge University Press, Cambridge, England, 1991).

[42] V. N. Popov, Quantum vortices and phase transitions in Bose systems, J. Exp. Theor. Phys. 37, 341 (1973).

[43] K. G. Wilson, Quarks and strings on a lattice, in New Phenomena in Subnuclear Physics: Part A, edited by A. Zichichi (Springer US, Boston, 1977), pp. 69-142.

[44] H. J. Rothe, Lattice Gauge Theories, World Scientific Lecture Notes in Physics Vol. 82 (World Scientific, Singapore, 2012).

[45] Even though we call it an area, it can have a negative value.

[46] I. Todhunter, Spherical Trigonometry, for the use of Colleges and Schools: With Numerous Examples (MacMillan, London, 1863).

[47] M. D. Frank-Kamenetski and A. V. Vologodski, Topological aspects of the physics of polymers: The theory and its biophysical applications, Sov. Phys. Usp. 24, 679 (1981).

[48] G. Călugăreanu, Lintégrale de Gauss et lanalyse des nœuds tridimensionnels, Rev. Math. Pures Appl 4, 5 (1959).

[49] G. Călugăreanu, Sur les classes d'isotopie des noeuds tridimensionnels et leurs invariants, Czech. Math. J. 11, 588 (1961). 
[50] J. H. White, Self-linking and the Gauss integral in higher dimensions, Am. J. Math. 91, 693 (1969).

[51] I. Montvay and G. Münster, Quantum Fields on a Lattice, Cambridge Monographs on Mathematical Physics (Cambridge University Press, Cambridge, England, 1994).

[52] C. R. Gattringer, S. Jaimungal, and G. W. Semenoff, Loops, surfaces and Grassmann representation in two- and threedimensional Ising models, Int. J. Mod. Phys. A 14, 4549(1999).

[53] The absolute value of the eigenvalue is the largest.

[54] We do not need to add negative values of $n_{a}$, since we can control the sign of vorticity with the orientation of the vortex loop.
[55] J. Ambjørn, B. Durhuus, and T. Jonsson, A random walk representation of the Dirac propagator, Nucl. Phys. B330, 509 (1990).

[56] Note that the difference of the overall sign in the writhe is immaterial, since we sum over loops with every possible orientation, with the winding number $n_{j}=1$ precisely matching the fermionic one $Z_{F}$ in the continuum limit.

[57] B. D. Josephson, Relation between the superfluid density and order parameter for superfluid He near $T_{C}$, Phys. Lett. 21, 608 (1966). 